\title{
Mutations du gène APC et instabilité chromosomique
}

L'analyse génétique des cancers colorectaux a permis de révéler l'existence de deux formes distinctes. La première, retrouvée dans $15 \%$ des cancers, est associée à un défaut du système de réparation des mésappariements de l'ADN qui se traduit par une instabilité des microsatellites (phénotype $\mathrm{MSI}^{+}$ou RER ${ }^{+}$) (revue dans [1]). La seconde, de loin la plus fréquente, puisque retrouvée dans $85 \%$ des cancers colo-rectaux, se caractérise par une instabilité chromosomique (phénotype $\left.\mathrm{LOH}^{+}\right)$. L'origine de cette instabilité chromosomique n'était jusqu'à présent pas clairement identifiée, mais deux articles récents montrent que les mutations de la protéine APC (adenomatous polyposis coli) pourraient bien en être responsables.

\begin{abstract}
APC: un partenaire essentiel de la voie de signalisation Wnt

Les lecteurs de médecine/sciences connaissent bien le rôle essentiel que joue APC dans la carcinogenèse colorectale. Des mutations inactivatrices du gène $A P C$ sont responsables d'une des deux formes de prédisposition familiale au cancer colorectal, la polypose adénomateuse familiale, et de $80 \%$ des formes sporadiques de cancer du côlon ([2] et $\mathrm{m} / \mathrm{s} 1995$, $n^{\circ} 3, p$. 443).
\end{abstract}

La protéine APC joue un rôle majeur dans le contrôle de l'activité de la voie de signalisation Wnt. Cette voie aboutit à la formation du complexe de transactivation $\beta$-caténine-TCF4, qui active la transcription de nombreux gènes cibles, en particulier l'oncogène $\mathrm{c}-M Y C$ ou la cycline $\mathrm{D} 1$. La protéine APC favorise la dégradation de la $\beta$-caténine au sein d'un complexe contenant aussi l'axine et la glycogène synthase kinase $3 \beta$. La principale conséquence des mutations inactivatrices d'APC est l'accumulation de $\beta$-caténine dans le cytosol et le noyau, et donc l'activation de la voie Wnt $\left(\mathrm{m} / \mathrm{s}\right.$ 1997, $n^{\circ} 6-7$, p. 872). S'il est bien établi qu'APC a tumeur, il semble cependant que ce ne soit pas sa seule fonction. En effet, la protéine APC peut se lier, par sa partie carboxy-terminale, aux microtubules, soit directement, soit par l'intermédiaire de EB1, une protéine qui est elle-même associée à l'extrémité positive des microtubules. Ceci suggérait donc que APC puisse aussi jouer un rôle dans l'organisation du cytosquelette ou dans celle du fuseau mitotique.

\section{APC, microtubules,} et instabilité chromosomique

Deux équipes décrivent en effet dans Nature Cell Biology que des cellules embryonnaires de souris homozygotes pour la mutation Min du gène $A p c$ acquièrent, au cours des passages en culture, des anomalies du nombre de chromosomes, qui sont liées à un défaut de ségrégation des chromosomes $[3,4]$.

L'allèle $A p c^{\min }$ code pour une protéine tronquée de sa partie carboxyterminale, qui ne peut alors ni se lier aux microtubules, ni participer à la dégradation de la $\beta$-caténine. D'autres mutations, comme la mutation $A p c^{1638 T}$, située dans une région plus distale, n'altèrent pas la fonction de dégradation de la $\beta$-caténine, mais provoquent aussi une instabilité chromosomique. Ceci suggère que cette instabilité n'est pas liée à un effet transcriptionnel induit par le complexe $\beta$-caténine-TCF4 [3]. Les deux groupes observent que la protéine APC native est localisée, pendant la mitose, à l'extrémité positive des microtubules, au niveau des kinétochores. Il s'agit probablement d'un attachement physique de la protéine APC à l'extrémité des microtubules, car leur dépolarisation empêche cette localisation. La protéine mutée $\mathrm{APC}^{\mathrm{min}}$ est, elle, incapable de se lier correctement aux kinétochores. Il existe alors une désorganisation du fuseau mitotique, de nombreux microtubules ne s'attachant plus aux kinétochores, et on observe parfois des centrosomes surnuméraires, ces anomalies étant très certainement à l'origine de l'instabilité chromosomique.

L'interaction entre les microtubules et la protéine APC se ferait par l'intermédiaire de la protéine EB1, partenaire connu de la protéine APC, dont la localisation normale aux niveaux des kinétochores disparaît dans les cellules exprimant la protéine APC mutée [3]. Les deux équipes émettent l'hypothèse d'un rôle de la protéine APC dans la stabilisation des microtubules et leur attachement aux chromosomes. En outre, APC interagit avec d'autres protéines du kinétochore, dont la protéine kinase BUB1 qui intervient dans le point de contrôle mitotique [4] $\left(m / s\right.$ 2001, $\left.n^{\circ} 3, p .353\right)$. Cependant, la signification de cette interaction n'est pas connue.

La fonction de la protéine APC n'est donc pas restreinte à celle de gène suppresseur de tumeur, mais pourrait aussi être essentielle à une ségrégation correcte des chromosomes lors de la mitose. Son altération pourrait ainsi rendre compte de l'aneuploïdie observée lors de la transformation maligne des cellules.

1. Laurent-Puig $\mathrm{P}$, Blons $\mathrm{H}$, Cugnenc $\mathrm{PH}$. Sequence of molecular genetic events in colorectal tumorigenesis. Eur J Cancer Prev 1999; 8: S3947.

2. Thomas G. Dix ans de recherche sur les prédispositions génétiques au développement des tumeurs. Med Sci 1995; 11 : 336-48.

3. Fodde R, Kuipers J, Rosenberg C, et al. Mutations in the APC tumor suppressor gene cause chromosomal instability. Nat Cell Biol 2001; 3 : 433-8.

4. Kaplan KB, Burds AA, Swedlow JR, Bekir SS, Sorger PK, Nathke IS. A role for adenomatous polyposis coli protein in chromosome segregation. Nat Cell Biol 2001 ; 3 : 429-32.

\section{Pierre Laurent-Puig} Hélène Blons

Laboratoire de toxicologie moléculaire, 45, rue des Saints-Pères, 75006 Paris, France. 